\title{
How to Avoid Artifacts in Nanobeam Diffraction Strain Measurements
}

\author{
B. Fuํ․ M. Gribelyuk ${ }^{1}$, Frieder H. Baumann ${ }^{1}$, Yun-yu Wang ${ }^{2}$ \\ 1. GLOBALFOUNDRIES, 400 Stone Break Rd Extension, Malta, NY, USA 12020 \\ 2. GLOBALFOUNDRIES, 2070 Route 52, Hopewell Junction, NY 12533
}

Strain engineering is indispensable in modern semiconductor manufacturing to increase the carrier mobility and enhance transistor performance. The precise measurement of the localized strain distribution near the transistor channel region is very critical to understand device performance. Among several experimental strain measurement techniques, nanobeam diffraction (NBD) is the most userfriendly method with good spatial resolution, large field-of-view, and good accuracy and precision [1]. However, the measurement results in NBD are strongly affected by experimental conditions and post data processing. In this study, the strain in an epitaxial grown SiGe monitoring pad is studied. The composition of the SiGe has been previously characterized by STEM-EDS [2]. The strain in [220] direction is expected to be $\sim 0$, as confirmed by dark field holography [3], shown in figure 1 .

In NBD experiments, an electron beam with small beam convergence angle is desired for improved measurement precision, figure 2(h). However, due to diffraction limit, the measurement spatial resolution is deteriorated with decreasing electron beam convergence angle, figure 2(a). Therefore, the experimentalist often optimizes the experimental conditions by trading-off the required spatial resolution and measurement precision. Another important, but often neglected quantity is the measurement accuracy. For the above-mentioned sample, NBD measurements with various beam convergence angles are performed. The strain is determined [4] by comparing the acquired diffraction patterns with reference pattern taken from the Si substrate. As shown in figure 2(b) and 2(c), the strain (relative lattice constant change) in [004] direction does not change with convergence angles. However, it is interesting to note that there is an obvious artificial negative strain measured in [220] direction, as the beam convergence angle increases. An analysis of the diffraction pattern, figure 2(d) and 2(e), shows such a measured artificial negative strain can be due to the dynamic contrast variation in reflection spots. This is often enhanced by auto-correlation techniques used in strain calculation software to improve the S/N and leads to the wrong determination of the diffraction spots' center. To overcome this problem, the data is pre-processed to find the real center of each diffraction disk [5] before calculating the strain. The postprocessing results, figure 2(f) and figure 2(g), shows the artifacts are removed and $\sim 0$ strain in [220] direction is measured. This is consistent with dark field holography results in figure 1.

To further confirm experimentally that the dynamic diffraction contrast variations in the reflections cause the measurement artifacts, precession enhanced electron diffraction (PED) [6] was used to measure the strain for the same sample. As shown in figure 3, a precession angle of only 0.5 degree is big enough to suppress the dynamic contrast effectively from the reflections in the diffraction patterns, and the measured negative strain artefacts in [220] direction are completely removed. These results show dynamic diffraction contrast variations in reflection disks can severely affect strain measurement accuracy in NBD, and two possible solutions to overcome this artifact are proposed. 
References:

[1] A. Béché et al, Applied Physics Letters 95 (2009), p.123114.

[2] B. Fu et al, Microsc. Microanal. 22(Suppl 3) (2016), p. 1618.

[3] M. Hytch et al, Nature 453 (2008), pp. 1086.

[4] F. H. Baumann, Applied Physics Letters 104 (2014), p.262102.

[5] The image processing using Python scripts includes: using the direct center spot in the diffraction patterns as a template and applying an edge filter to find real center of all the diffraction disks.

[6] R.Vincent et al, Ultramicroscopy 53 (1994), p. 271.
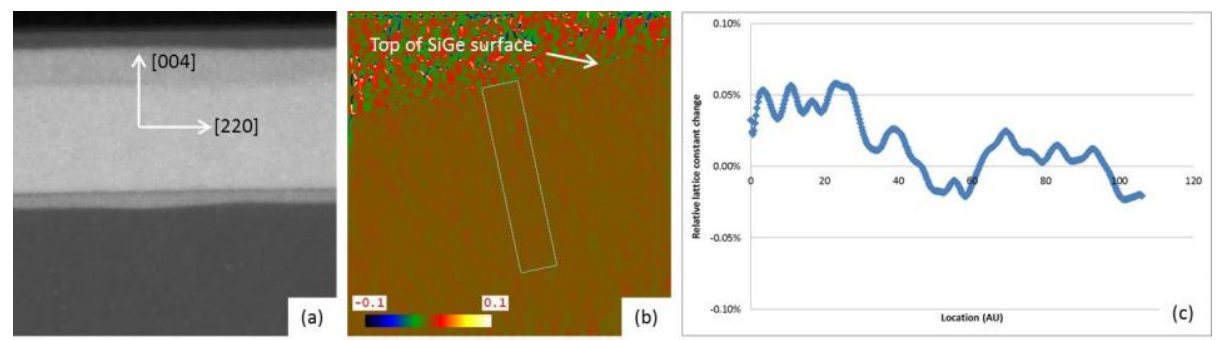

Figure 1. (a) STEM image of the epitaxial SiGe pad; (b) Strain map in [220] direction measured by dark field holography; (c) Line scan from box in (b), showing the negligible strain in [220] direction.
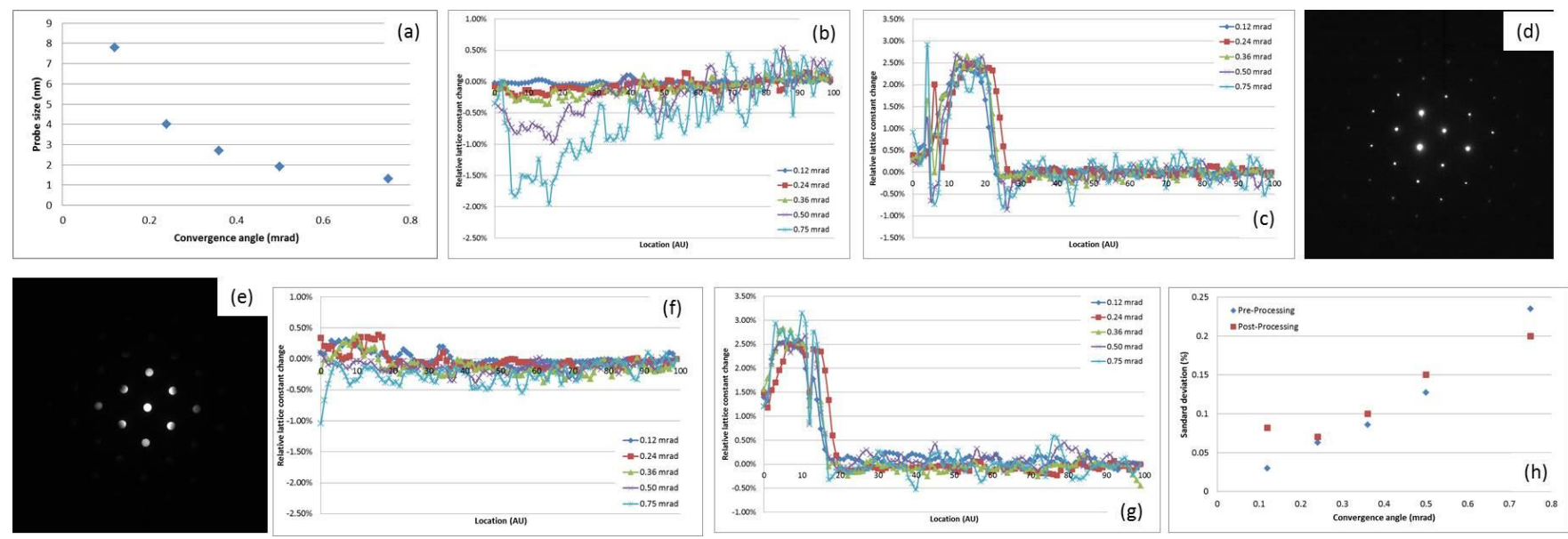

Figure 2. (a) Measured probe sizes with various electron beam convergence angles; NBD strain measurements with various convergence angles at [220] direction (b) and [004] direction (c) prior to image processing and at [220] direction (f) and [004] direction (g) after image processing; Example diffraction patterns at convergence angles $0.12 \mathrm{mrad}(\mathrm{d})$ and $0.75 \mathrm{mrad}(\mathrm{e})$; (h) Strain measurement noise level in $\mathrm{Si}$ substrate with various electron beam convergence angles prior-to and after image processing.
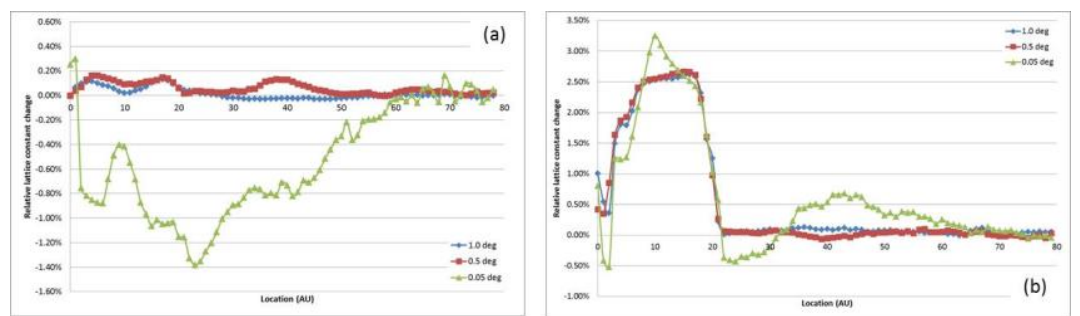

Figure 3. Precession enhanced electron diffraction (PED) measurements of the SiGe strain with various precession angles at [220] (a) and [004] (b) directions. 